\title{
Cryptocurrency price prediction based on multiple market sentiment
}

\author{
Yu Wang \\ Renmin University of China \\ 18810920366@163.com
}

\author{
Runyu Chen \\ Renmin University of China \\ ry.chen@,ruc.edu.cn
}

\begin{abstract}
With the rapid development of the Internet, cryptocurrencies have been gaining increasing amounts of attention dramatically. As a digital currency, it is not only used worldwide for online payments, but also traded as an investment tool on the market. Therefore, the ability to predict the price volatility will facilitate future investment and payment decisions. However, there are many uncertainties in the price movement of cryptocurrencies, and the prediction is extremely difficult.

To this end, based on the transaction data of three different markets and the number and content of user comments and responses from online forums, this paper constructs a price prediction model of cryptocurrencies using a variety of machine learning and deep learning algorithms. It turns out that the trading price premium rate in different markets will affect the price to be predicted, and adding social media comment features can significantly improve the accuracy of the forecast. This article is conducive to investors who encrypt currencies to make more scientific decisions.
\end{abstract}

\section{Introduction}

The rapid development of internet has triggered the emergence of cryptocurrencies, which is a new asset class. Recently, it has been gaining increasing amounts of attention dramatically, since the introduction of the media, finance industry and academics [1]. There are two main reasons. First, the market size of cryptocurrency is increasing rapidly. According to Aicoin data, the total market capital of the cryptocurrency market hit $\$ 50$ billion in 2017 and over $\$ 140$ billion at the start of 2019. Besides, more and more countries have recognized that cryptocurrency is a legitimate payment method. Bitcoin has been created in 2009 by Nakamoto, which ranks first in the cryptocurrency market, following by Ethereum and Ripple [2].

Cryptocurrencies such as Bitcoin assume the role of digital currency. It is not only used worldwide for online payments, but also traded as an investment tool on the market [3]. Therefore, the ability to predict the price volatility of Bitcoin will facilitate payment and investment decisions. However, as a form of value storage, the price fluctuation of cryptocurrency is uncertain. There are salient differences between cryptocurrencies and traditional currency in terms of price fluctuation [4]. Hence, price prediction of the former is extremely difficult. In recent years, scholars have also been increasingly focused on the price forecast of cryptocurrency. However, most of them perform the experiment with traditional price time series data and regression models. Stated differently, the research of cryptocurrency price prediction is still at an initial stage.

Therefore, the objective of the present paper is to establish a more accurate cryptocurrency price prediction model. One novelty of our research is that we collect data from multiple markets, such as fiat, exchange and contract trading market. The addition of trading data from diverse markets makes the predicted feature set richer. In addition, the price difference between multiple markets also reflects the price change to some extent. Therefore, considering multiple market sentiment can increase the accuracy of the forecast. Besides, we construct a sentiment lexicon of cryptocurrency to increase the accuracy of market sentiment evaluation. This sentiment dictionary is specifically for cryptocurrency and can be employed as a reference for future research on cryptocurrency related comments. Ultimately, we employ diverse machine learning and deep learning methods for prediction and find that LSTM has the highest accuracy.

\section{Related work}

Research on the price prediction of cryptocurrency has been extensively conducted. The initial study 
adopted a trading time series data to construct a price prediction model. In general, cryptocurrency trading data includes daily closing price and volume $[5,6]$. However, the price of the cryptocurrency from different exchanges is affected by various variables so that a price difference exists. These variables include market size, trading volume, entry price and currency of trade. Therefore, Brandvold et al. selected price, volume for each exchange and timestamp of seven exchanges to analyze price differences [7]. The results show that the two highest information share in the market are Mt.Gox and BTC-e.

However, from the perspective of actual cryptocurrency trading, the market can be divided into three parts: exchange trading, fiat trading and contract trading. The exchange trading is the transaction between digital currencies on the exchange. The fiat trading refers to the user's exchange of fiat and the digital currency through OTC (over-the-counter) transaction. Contract trading is a standardized contract based on the cryptocurrency. Previous studies only consider exchange trading, while other trading markets such as the fiat and contract trading markets will also cause price volatility. Therefore, this paper considers transaction data of multiple markets to predict the price of cryptocurrency.

Since cryptocurrency is broadly similar to financial assets and speculative assets that can be traded in financial markets, its price fluctuation is largely influenced by economic factors. Among the factors, the daily EPU index in the U.S., is widely considered to have a significant correlation to the bitcoin revenue [8]. Besides, Li and Wang explored the Bitcoin exchange rate determination by taking into consideration economic factors such as money supply, GDP, interest rate and inflation [9]. Other key economic indicators such as the US S\&P 500 index, the US dollar index futures, the RMB to US dollar Exchange rate, Stock Composite Index, the RMB to U.S. dollar exchange rate, the FTSE China A50 and the WTI crude oil are simultaneous available to the price fluctuation of cryptocurrencies [10].

In addition to relevant data of the financial market, along with the development of the Internet, social media such as the online community frequently discuss cryptocurrency transactions, and the resulting opinions and emotional sentiment can be used as an important factor in some investment decisions through word-ofmouth communication. The relationship between social media and stock price is inconsistent across prior studies. Similarly, introducing social media into cryptocurrency price forecasting has become a new trend. The study found that the number of user reviews and emotions on online forums or Twitter would cause fluctuations in cryptocurrency price. Positive comments and replies are found to be significantly associated with fluctuations in the price of Bitcoin. In addition, more bullish forum posts are associated with higher future bitcoin values $[11,12]$. Moreover, adding social media features can greatly increase the accuracy of price prediction $[5,13,14]$.

However, some scholars believe that online factors do not necessarily maintain a stable relationship with prices [2]. It is believed that the relationship between online factors and prices has been previously seen to appear and disappear overtime due to the existence of bubble-like regimes in the price of cryptocurrencies. Eventually, in the short term, online factors may not be the best predictors, while they tend to show a stronger and stable relationship in the long run. In addition, It is found that Google's page views of relevant information can also increase the price forecasting ability of cryptocurrencies $[10,15]$.

Traditional price prediction models usually adopt econometric models, the most widely used of which are multivariate linear regression, GARCH model, extended Vector Autoregressive model and multivariate vector autoregressive model [2, 16, 17, 18, $19,20]$. With the development of big data technology and artificial intelligence, a growing body of empirical studies has applied machine learning models to classification and prediction. In the financial industry, the application and practice of machine learning algorithms in stock price forecasting is relatively mature [5]. Subsequently, scholars try to conduct machine learning models for blockchain research. For example, Kim et al. adopted machine learning models to mine user reviews and found that the number of responses to user reviews and online comments affects the number of transactions between users [12]. Peng et al. constructed the predictive model by combining SVR and GARCH model [3]. Finally, the performance of SVR-GARCH model is better than GARCH, EGARCH and GJR-GARCH models. Other scholars compared artificial neural network (ANN), support vector machine (SVM) and set algorithm based on recurrent neural network and k-means clustering method to predict the price of daily Bitcoin exchange rate. In summary, it is found that the application of machine learning algorithms can significantly improve the accuracy of predictive models $[19,21]$. Therefore, this paper combines transaction data from multiple markets and social media data and applies a variety of machine learning methods to construct a price prediction model for cryptocurrencies.

\section{Methods}

\subsection{Framework}


The research framework of this paper is shown in figure 1 . The process can be divided into three parts, data collection and processing, model construction and model evaluation.

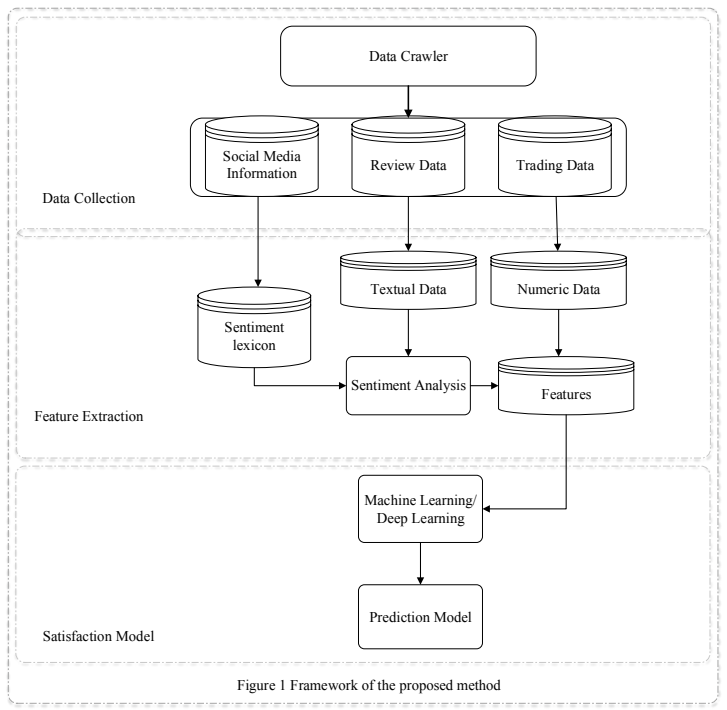

Figure 1. Framework of the proposed method

The first step is data crawling. This paper performs python's selenium module to crawl user comment data about cryptocurrencies on the online forums. In addition, we also obtained market trading data from two famous cryptocurrency exchanges in China. In detail, market data includes daily exchange transaction data, fiat transaction data, and contract transaction data. More importantly, we divided the collected data into two parts, training data and test data. The former is used to train the cryptocurrency price prediction model, while the latter is employed to verify the validity and verification of our proposed model before. At the same time, in order to expand sentiment lexicon, data from social media like Weibo was also collected.

The second step is sentiment recognition. The collected data can be divided into two types: text data and numeric data. Among them, the market trading data is numerical and easy to process. However, the user comment is text type data, and each comment needs to be converted into an emotion value through sentiment analysis, so as to be added as a feature to the prediction model. In this step, the sentiment lexicon is employed to mine topics and sentiment contained in the comments. To this end, we have collected social media which is relevant to cryptocurrency, and built a sentiment lexicon for cryptocurrency based on HowNet to improve the accuracy of sentiment analysis.

The third step is Model Construction. This paper selects a variety of machine learning and deep learning algorithms for prediction, including LSTM, CNN,
SVM, BPNN, RBF and compares the prediction models to find the best one.

The final step is model evaluation. This paper compares the accuracy of different algorithm models with MAPE, MAE, and RMSE.

\subsection{Data collection and analysis}

The data source of this paper is divided into two parts, one is to obtain the transaction data of the market through the API of the exchange database, and the other is to obtain the relevant user comments of the cryptocurrency corresponding to the time through the web crawler. This article collects data for the first quarter of 2019. This includes transaction data for three markets, and the cryptocurrency transaction is $24 \mathrm{~h}$ uninterrupted. In detail, we collected data for five currencies in OTC, currency and contract market, namely Bitcoin (BTC), Ethereum (ETH), Tether USD (USDT), EOS, Ripple (XRP), litecoin (LTC). The data items included are time, the highest price, the lowest price, opening price, closing price, volume and currency. Accordingly, we crawled daily user reviews on online forums from the third quarter of 2018 to the first quarter of 2019. The forum has different communities set up for different cryptocurrencies. We perform python to crawl the source code of each community's HMTL and match the corresponding data with regular expression. Specific data items include the content and quantity of title, comments, replies, and time of publication.

In addition, we have preprocessed data from two different sources. For market trading data, in addition to the price time series data of different currencies, this paper believes that the spread premium existing in different markets will also affect the price to be predicted. To this end, this paper calculates the spread of exchange and fiat market and the spread of contract and exchange market. The spread premium rate can be calculated as:

$$
\text { Premium_rate }=\frac{\left(P_{a}-P_{b}\right)}{P_{b}}
$$

Where $a$ and $b$ are the type of market, for example, when a indicates the fiat market, $b$ indicates the exchange market, then $\mathrm{Pa}$ represents the transaction price of fiat market, while $\mathrm{Pb}$ represents the transaction price of exchange market. And their difference divided by the price of the exchange market is the ratio of the premium. In addition, $\mathrm{Pa}$ can also represent the trading price of contract market. The premium ratios in different markets can reflect certain market sentiment. The larger the value, the greater the mood swings in the market, which inevitably affects trading price. 


\subsection{Sentiment identification}

In addition to market trading data, a large number of user comments on social media are also reflected in the price of cryptocurrencies through effects such as word-of-mouth communication. To this end, we employ the model of sentiment analysis to dig out emotions in diverse cryptocurrencies from user comments. Sentimental analysis is people's perception, emotions, and assessments of products, services, organizations, and so on. In order to judge the consumer's perception of the attitude of cryptocurrency, sentiment analysis can be performed on the comment, and the emotional value of consumers is expressed by the magnitude of the emotional value. If the sentiment value is negative, it indicates that the consumer's attitude towards cryptocurrency is negative, which means that people are generally not optimistic about this price, and there may be a large amount of selling of cryptocurrency in the future. Therefore, it will cause a large price drop. We apply a sentiment lexicon based approach to calculate the emotional value of each comment. Here, we mainly adopt HowNet lexicon. In order to accurately determine the sentiment of comments, this article specifically crawl a large amount of comment data with keywords related to cryptocurrency. Before adding emotional words to the open-source sentiment lexicon, we extract them which are commonly used by users to comment on cryptocurrency. Finally, we construct a sentiment lexicon specifically for cryptocurrency reviews. The specific method is to add the emotional seed word through calculating the mutual information and entropy between seed word and each word in the corpus. After that we combine the mutual information with entropy to select the Top $\mathrm{N}$ words with the highest degree of relevance to seed words and add them to the corresponding sentiment lexicon.

The formula for calculating the mutual information is as follows:

$$
P M I(x, y)=\log _{2} \frac{p(x, y)}{p(x) p(y)}
$$

In the above formula, $\mathrm{p}(\mathrm{x}, \mathrm{y})$ is the probability that two words appear together, $\mathrm{p}(\mathrm{x})$ is the probability of occurrence of the word $x$, and $p(y)$ is the probability of the word y appearing.

By mutual information calculation, words with a relatively large correlation with known words in the lexicon can be found. In addition, we also use entropy to measure the degree of freedom of the pre-selected words. The greater the entropy, the more words are converted to the left of the word, so it is likely to be a single word. Take the left entropy as an example, it is defined as:

$$
E_{L}(W)=-\sum_{\forall a \subseteq A} P(a W \mid) \log _{2} P(a W \mid W)
$$

Where $\mathrm{W}$ is the pre-selected word and $\mathrm{A}$ is the word that appears to the left of the pre-selected word.

\subsection{Prediction model}

We apply a variety of machine learning algorithms to predict the price of cryptocurrencies, including BP neural network (BPNN), RBF, support vector machine (SVM), and deep learning algorithm CNN, LSTM. The price prediction model is evaluated by comparing the accuracy of the five algorithms with the evaluation indicators. We adopt python's sklearn module to implement RBF, BPNN and SVM, while CNN and LSTM use the tensorflow framework.

\section{Empirical analysis}

\subsection{Data description and evaluation criteria}

The trading data are derived from the actual values of the two largest cryptocurrency trading platforms in China, binance and huobi. They provide trading data for different cryptocurrencies in the OTC, exchange and contract market from January to March 2019. We made predictions regarding five crytocurrencies, Bitcoin (BTC), Ethereum (ETH), Tether USD (USDT), EOS, Ripple (XRP), litecoin (LTC). In the first quarter, the transaction data of the two exchanges were 820994 and 1008163 respectively. Table 1 outlines the arrangement of the market data that were gathered.

Table 1. Market data of cryptocurrencies

\begin{tabular}{lll}
\hline \multirow{2}{*}{ Exchange } & \multicolumn{2}{l}{ Cryptocurrency prices } \\
\cline { 2 - 3 } & Crawling Boundary & Data volume \\
\hline binance & Jan. 01, 2019- & 820994 \\
huobi & Mar.31, 2019 & 1008163 \\
\hline Exchange & Cryptocurrency transactions \\
\cline { 2 - 3 } & Crawling Boundary & Data volume \\
\hline $\begin{array}{l}\text { binance } \\
\text { huobi }\end{array}$ & Jan. 01, 2019- & 820994 \\
& Mar.31, 2019 & 1008163 \\
\hline
\end{tabular}

Among them, the transaction price descriptions of different markets are shown in the following table 2 . Here, we take the average of each type of price.

Table 2. Data description of market price

\begin{tabular}{llll}
\hline & $\begin{array}{l}\text { Exchange } \\
\text { trading } \\
\text { market }\end{array}$ & $\begin{array}{l}\text { Fiat } \\
\text { trading } \\
\text { market }\end{array}$ & $\begin{array}{l}\text { Contract } \\
\text { trading } \\
\text { market }\end{array}$ \\
\hline high & 10709.36 & 12138.49 & 10998.01 \\
open & 10678.82 & 10523.63 & 10995.96 \\
close & 10683.91 & 10132.1 & 10980.41
\end{tabular}


low

10653.37

8134.32

10979.01

In addition, this article crawled user comment data from online forums. As mentioned above, the sentiment of comment has a predictive effect on cryptocurrency price, and there is a certain lag. Therefore, we determine the crawling boundary of the comment data based on the date range of the transaction data. In the end, this article crawled the user review data from December 2018 to the first quarter of 2019. Table 3 describes the crawled comment information data.

Table 3. Comment data of Cryptocurrencies

\begin{tabular}{|l|l|l|}
\hline \multirow{2}{*}{ Cryptocurrency } & \multicolumn{2}{|l|}{ Comment data } \\
\cline { 2 - 3 } & Source & Data volume \\
\hline BTC & BTC Forum & 13892 \\
\hline ETH & ETH Forum & 2578 \\
\hline USDT & USDT Forum & 689 \\
\hline EOS & EOS Forum & 540 \\
\hline XRP & Ripple Forum & 622 \\
\hline LTC & LTC Forum & 458 \\
\hline
\end{tabular}

As mentioned above, there are two types of data that can be used to train and test the validity and characteristics of the model. The first is market trading data, which includes the highest price, lowest price, opening price, closing price, volume, currency for three markets. Besides, the premium rate of the fiat and exchange markets and the premium rate of the contract and exchange market are also considered. The second category is user comment data processed by sentiment analysis, including title, comments, sentiment, quantities of the replies, and publication time. During model training, the data is divided by day. Diverse lags of review data according to previous studies may have an impact on the current price.

To evaluate the effectiveness of different prediction models, two kinds of indicators are adopted as evaluation criteria. One is root-mean-square-error (RMSE), which measures the deviation of the predicted value from its true value. Mean absolute difference (MAE), which better reflects the actual situation of the predicted value error. The formulas are as follows:

$$
R M S E=\sqrt{\frac{\sum_{i=1}^{m}\left(a_{i}-p_{i}\right)^{2}}{\mathrm{~m}}}
$$

$$
M A E=\frac{\sum_{i=1}^{m}\left|a_{i}-p_{i}\right|}{m}
$$

Where ${ }^{a_{i}}$ represents the actual emotional value and $p_{i}$ represents the emotional value predicted by algorithms.

\subsection{Experimental results}

Based on the designed experiments mentioned in section 3, five types of models are employed to predict the cryptocurrency prices. For every model, there are two kinds of input: one is transaction data from diverse markets and the other adds the features, the sentiment and quantity of comments and responses by users.

In order to compare the effectiveness of different features, we designed two experiments to predict with diverse features. The first one is historical trading data with only three markets and the premium rate among different markets, which is marked as 1 in the experiment. The second one marked as 2 adds the sentiment of comments on the basis of the previous one. In each experiment, in addition to considering the impact of the current eigenvalues, their historical value may also affect the current price. For example, when people trade cryptocurrencies, they usually browse comments made by users before, which may be published for a long time.

In the experimental session, five most widely used machine learning and deep learning algorithms are invoked to predict the price of cryptocurrencies. As mention above, two neural network algorithms, BPNN and RBF are performed. Besides, we choose an important branch of SVM (Support Vector Machine) specifically for regression, SVR (Support Vector Regression). Two different kernel functions are selected, which are Linear and Sigmoid function. The reason is that the fitting effect of other kernel functions is not good. In addition, we also selected two deep learning algorithms, $\mathrm{CNN}$ and LSTM. In order to compare the validity of the model, we compare two evaluation indicators of the five algorithms.

Experimental results based on standard MAE are shown in figure 2. Firstly, the MAE of experiment 2 is generally smaller than that of Experiment 1, which means the addition of the sentiment and quantitative features of reviews can make the prediction more accurate. Stated differently, the addition of social media data features can also increase the accuracy of predictions. In addition, we find that using the neural network algorithm, that is, BPNN and RBF are worse 
than the overall effect of SVR, while both indicators of LSTM and CNN are relatively small, indicating that the prediction accuracy is higher. Among the two deep learning algorithms, the LSTM performs better.

In detail, in terms of neural network models, the study find that RBF performs better than BPNN. However, the prediction accuracy of the two neural networks is not as good as the SVM and deep learning algorithms. Although SVM and deep learning algorithms take more time to learn and predict. For the SVR model, there are some differences in the error when selecting different kernel functions. The sigmoid kernel function is better than the linear kernel function under the same conditions of the correlation lag parameter, and the training time is relatively short. In terms of CNN and LSTM, their MAE is generally small. Compared with CNN, LSTM is a more standard time series data analysis, so it performs better when predicting prices. In general, the LSTM model considering two types of data features can perform best at the smallest MAE.

For different lags, 1 to 7 day's lag can lead to better predictions. The result is that when people conduct bitcoin transactions, they often pay attention to market sentiment in the past week, which affects their decision-making.

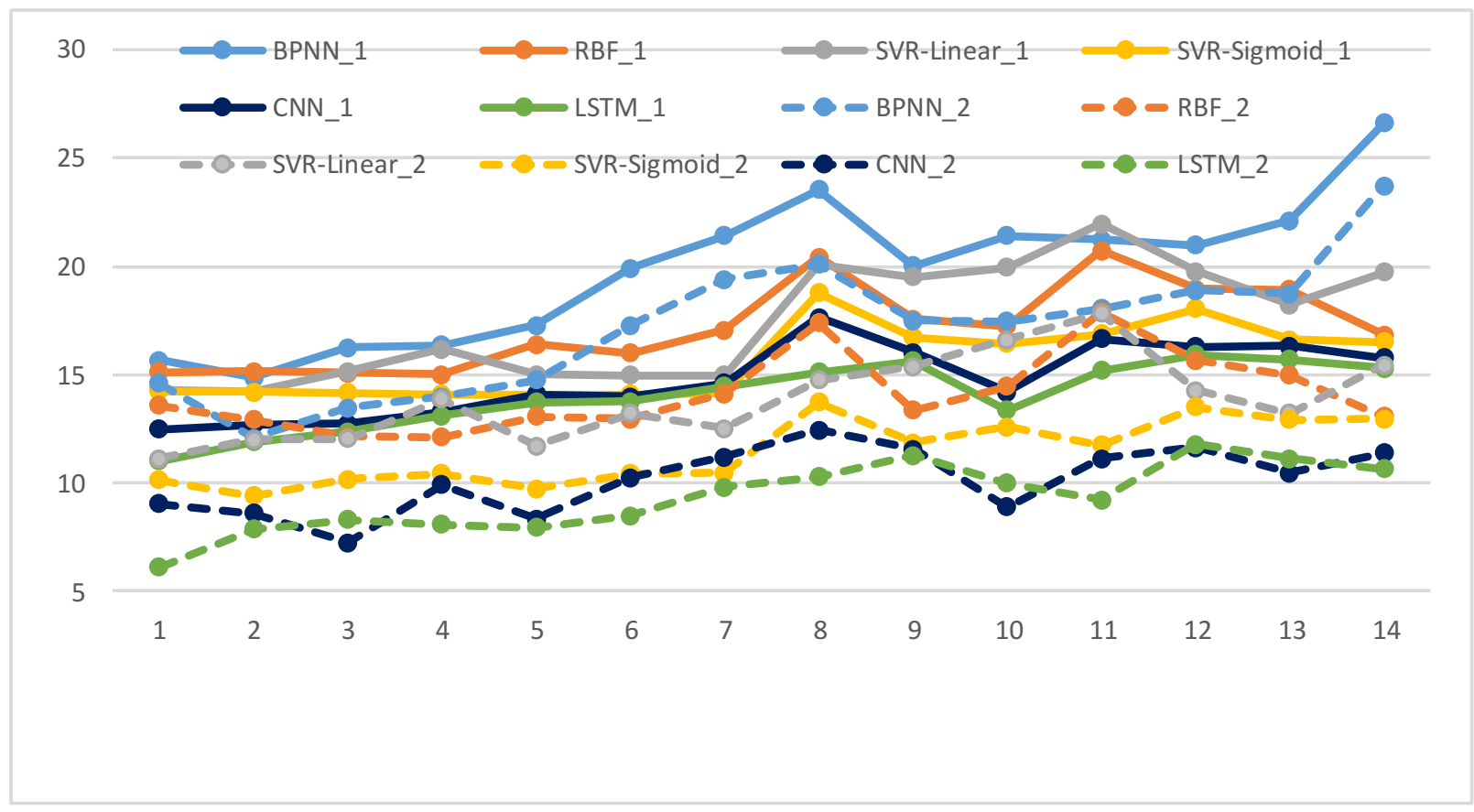

Figure 2. MAE of predictive models

Experimental results based on standard RMSE are shown in figure 3 . We can get similar results. Models with only historical price transaction data has worse results than that with two types of data features. Besides, LSTM model can get the best predictions with the smallest MAE. In terms of different lags, in addition to the SVR model, 1 to 7 day's lag in other models can also lead to better predictions. The results once again prove that the market sentiment expressed by user comments on social media for the past week will affect the decision-making of users.

To further explore the effects of price prediction, we experiment with separate datasets in different cryptocurrencies. Since the previous experiments proves that LSTM has the best prediction effect, we use it to perform two sets of experiments on each currency sample, and compare MAE and RMSE to judge the prediction effect of diverse cryptocurrencies. Figure 4 and 5 show RMSE of the prediction models for diverse cryptocurrencies. The results show that basically the prediction error of the second experiment for each currency was lower than that of the first group. In addition, the prediction error of BTC is smaller than that of other cryptocurrencies, and EOS has the biggest error. For different lags, the best predicted lag values for most cryptocurrencies are between 1 and 7 . Relatively speaking, after adding comments, the best predicted lag values of BTC, ETH, XRP and LTC show less trend, indicating that the addition of social media information facilitates consumers reduce the hesitation time. Specifically, figure 4 shows the value of the RMSE over time for four cryptocurrencies with reduced lags. Among them, Bitcoin had the best predicted lag value of 3 in the first experiment and 2 in 
the second. Similarly, the best predicted lag value of ETH changes from 4 to 2, the optimal predicted lag value of XRP changes from 2 to 1 , and the optimal predicted lag value of LTC changes from 5 to 4 . The best predicted lag value of USDT and EOS shows an increasing trend, that is, users will refer to more historical information when trading these two cryptocurrencies. This result can be seen from figure 5 , the best predicted lag value of USDT increases from 5 to 7 , and the optimal predicted lag value of EOS increases from 2 to 5 .

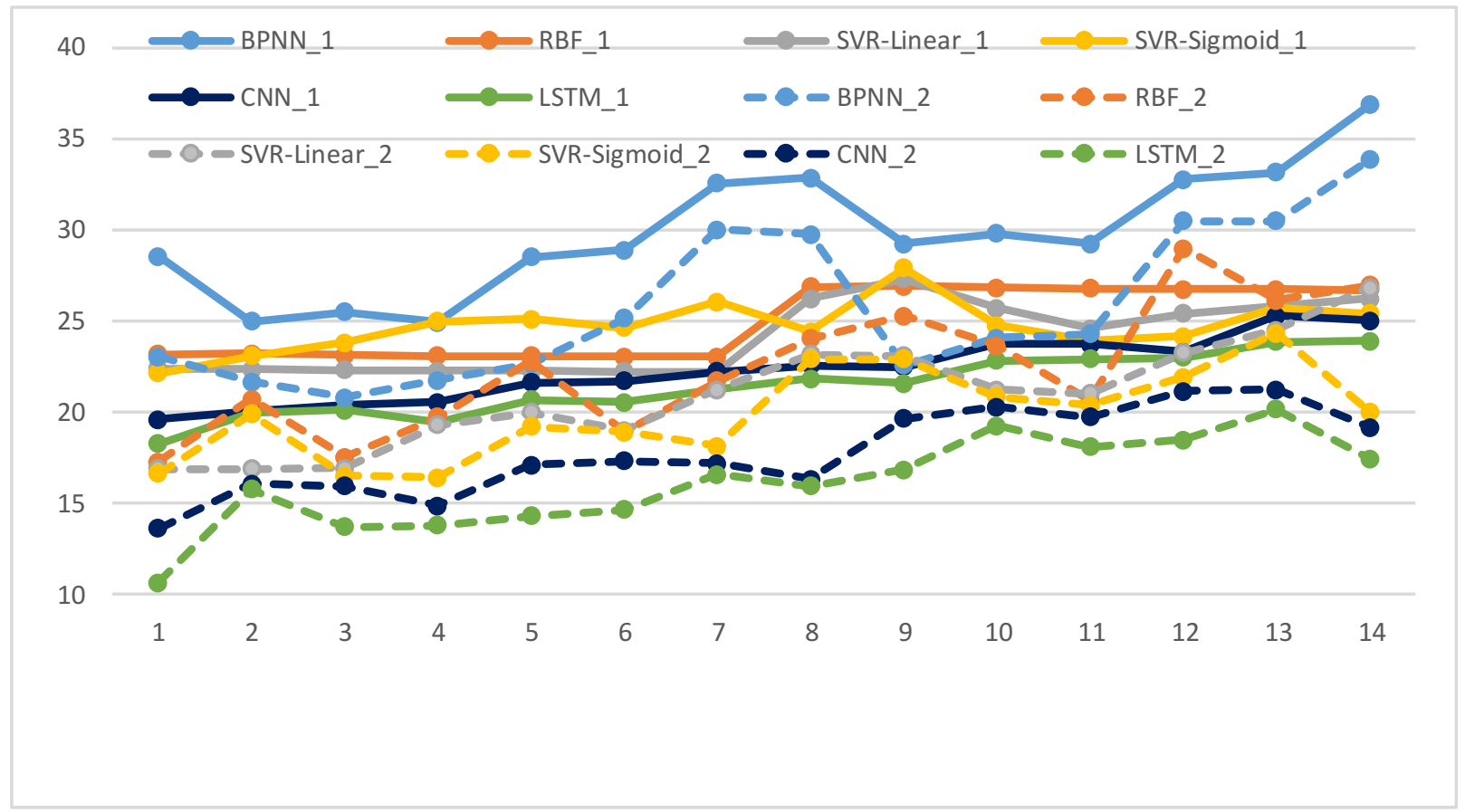

Figure 3. RMSE of predictive models

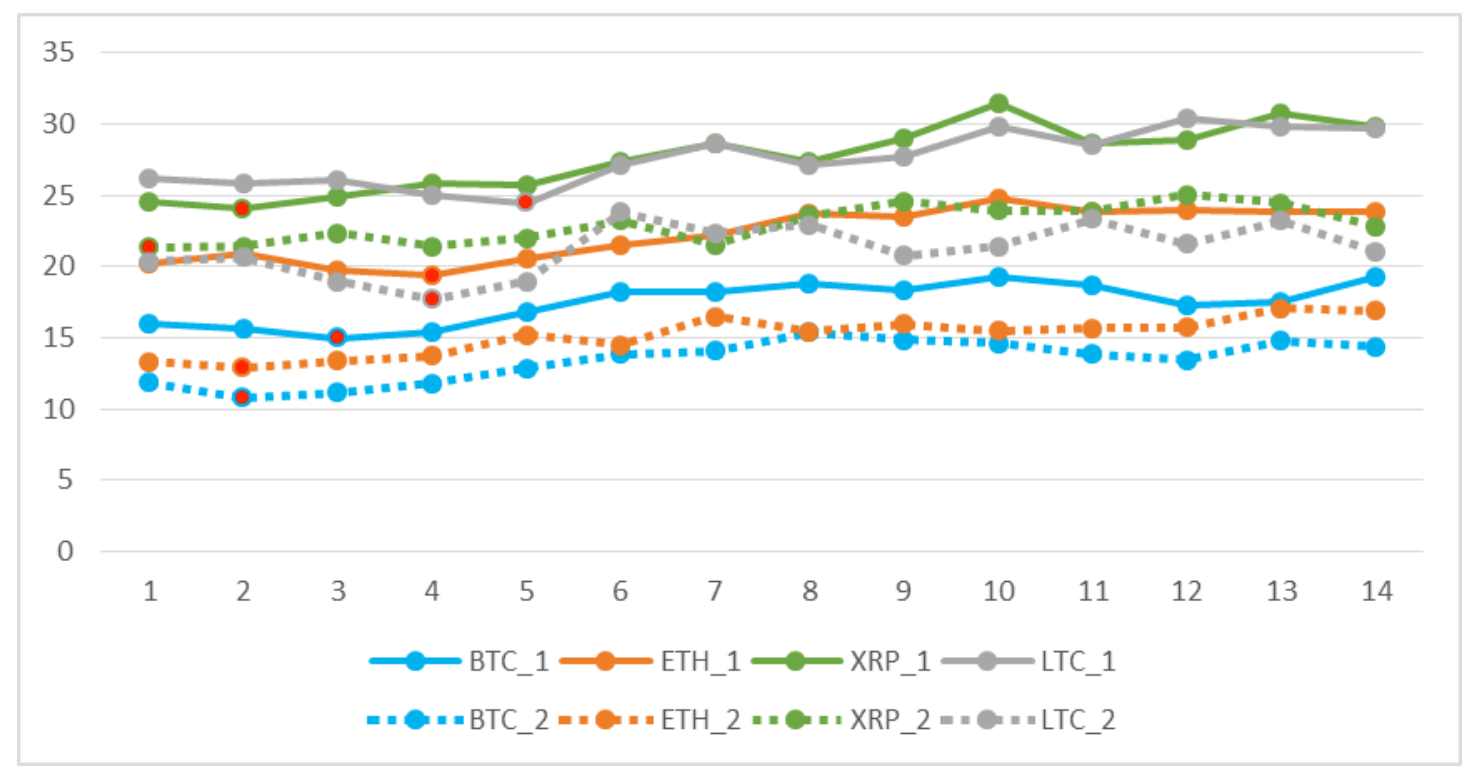

Figure 4. RMSE of predictive models for BTC, ETH, XRP, LTC 


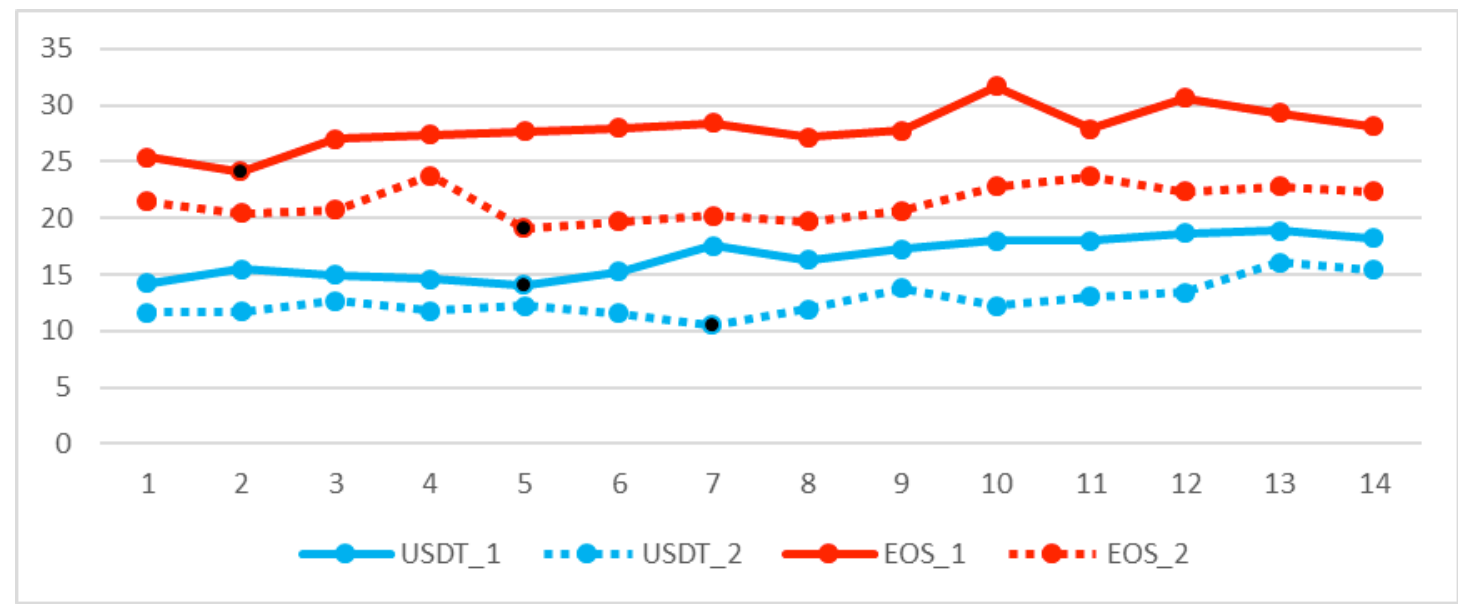

Figure 5. RMSE of predictive models for USDT, EOS

\section{Conclusion and future work}

This paper presents a price prediction model for cryptocurrency. Unlike previous studies, we considered the sentiment of different markets and the impact of social media sentiment on price forecasts. To this end, we collected transaction data in fiat, exchange and contract trading market. Besides, user reviews on forums in diverse cryptocurrencies are also crawled. Then we can divide these into transaction data and comment data. In the transaction data, in addition to all kinds of prices in each market, we also add price premium rates among different markets. In terms of comments, we specially construct a sentiment lexicon based on cryptocurrency, which quantify user comments into emotional values.

Moreover, we employ a variety of machine learning and deep learning methods to construct predictive models. The results show that the LSTM model has the best prediction effect, and the addition of comments' sentiment can significantly improve the accuracy of prediction. In addition, trading data and comment data that are 1-7 days before are the main reference for users to make trading decisions. More specifically, for different cryptocurrencies, the best predicted lag values for BTC, ETH, XRP, and LTC show less trend after adding review information, while those of USDT and EOS presents an increasing trend. The conclusions of this paper can not only provide theoretical and methodological guidance for future investment and payment decisions, but also provide some reference for the research of price forecasting of cryptocurrency.

In addition, there are some research questions in this study that require further research. First, some macroeconomic factors and other user behavior information (such as search volume) can be employed to further improve forecast performance. In addition, the amount of comment data in some cryptocurrencies selected in this paper is small, so that there may exist some errors in the forecasting process. In the following study, the experiment will be carried out with sufficient data volume. Finally, in the future, the cryptocurrency trading strategy system will be further developed to facilitate investors conduct price trend analysis.

\section{Acknowledgments}

This work was supported in part by the National Natural Science Foundation of China (Grant No. 71771212, U1711262), Humanities and Social Sciences Foundation of the Ministry of Education (No. 14YJA630075, 15YJA630068), and Fundamental Research Funds for the Central Universities, and Research Funds of Renmin University of China (No. 15XNLQ08). Mr. Chen's work is supported by the Outstanding Innovative Talents Cultivation Funded Programs 2017 of Renmin University of China. Runyu Chen is the corresponding author of this paper.

\section{References}

[1] Catania, L., Grassi, S., and Ravazzolo, F, "Forecasting cryptocurrencies under model and parameter instability", International Journal of Forecasting, 35(2), 2019, pp. 485501.

[2] Phillips, R. C., and Gorse, D., "Cryptocurrency price drivers: Wavelet coherence analysis revisited", PLOS ONE,13(4), 2018, pp. 
[3] Phillips, R. C., and Gorse, D., "Mutual-Excitation of Cryptocurrency Market Returns and Social Media Topics", arXiv: Social and Information Networks, 2018, pp. 80-86.

[4] Kim, K., Kim, J., and Rinaldo, A., "Time Series Featurization via Topological Data Analysis: an Application to Cryptocurrency Trend Forecasting", arXiv: Computational Geometry, 2018, pp.

[5] Phillips, R. C., and Gorse, D., "Predicting cryptocurrency price bubbles using social media data and epidemic modelling", IEEE Symposium Series on Computational Intelligence, 2017, PP, 1-7.

[6] Begusic, S., Kostanjcar, Z., Stanley, H. E., and Podobnik, B, "Scaling properties of extreme price fluctuations in Bitcoin markets", Physica A-statistical Mechanics and Its Applications, 2018, pp. 400-406.

[7] Brandvold, M., Molnar, P., Vagstad, K., and Valstad, O. $\mathrm{C}$, "Price discovery on Bitcoin exchanges. Journal of International Financial Markets", Institutions and Money, 2015, pp. 18-35.

[8] Demir, E., Gozgor, G., Lau, C. K., and Vigne, S. A, "Does economic policy uncertainty predict the Bitcoin returns? An empirical investigation", Finance Research Letters, 2018, pp. 145-149.

[9] Li, X., and Wang, C. A., "The technology and economic determinants of cryptocurrency exchange rates", Decision Support Systems, 95(95), 2017, pp. 49-60.

[10] Sun, X., Liu, M., and Sima, Z., "A novel cryptocurrency price trend forecasting model based on LightGBM", Finance Research Letters, 2018, pp.

[11] Mai, F., Shan, Z., Bai, Q., Wang, X., and Chiang, R. H, "How Does Social Media Impact Bitcoin Value? A Test of the Silent Majority Hypothesis", Journal of Management Information Systems, 35(1), 2018, pp. 19-52.

[12] Kim, Y. B., Kim, J. G., Kim, W., Im, J. H., Kim, T. H., Kang, S. J., and Kim, C., "Predicting Fluctuations in Cryptocurrency Transactions Based on User Comments and Replies", PLOS ONE, 11(8), 2016, pp.

[13] Kim, Y. B., Lee, J., Park, N., Choo, J., Kim, J. H., and Kim, C., "When Bitcoin encounters information in an online forum: Using text mining to analyse user opinions and predict value fluctuation", PLOS ONE, 12(5), 2017, pp.

[14] Shen, D., Urquhart, A., and Wang, P., "Does Twitter predict Bitcoin. Economics Letters", 2019, pp. 118-122.

[15] Brandvold, M., Molnar, P., Vagstad, K., and Valstad, O. C., "Price discovery on Bitcoin exchanges", Journal of International Financial Markets, Institutions and Money, 2015, pp. 18-35.
[16] Atsalakis, G. S., Atsalaki, I. G., Pasiouras, F., and Zopounidis, C., "Bitcoin price forecasting with neuro-fuzzy techniques", European Journal of Operational Research, 276(2), 2019, pp. 770-780.

[17] Begusic, S., Kostanjcar, Z., Stanley, H. E., and Podobnik, B., "Scaling properties of extreme price fluctuations in Bitcoin markets", Physica A-statistical Mechanics and Its Applications, 2018, pp. 400-406.

[18] Lahmiri, S., and Bekiros, S., "Cryptocurrency forecasting with deep learning chaotic neural networks", Chaos Solitons \& Fractals, 2019, pp. 35-40.

[19] Aalborg, H. A., Molnar, P., and De Vries, J. E., "What can explain the price, volatility and trading volume of Bitcoin?", Finance Research Letters, 2018, pp.

[20] Giudici, P., and Abuhashish, I., "What determines bitcoin exchange prices? A network VAR approach", Finance Research Letters, 2019, pp. 309-318.

[21] Shynkevich, Y., Mcginnity, T. M., Coleman, S. A., and Belatreche, A., "Forecasting movements of health-care stock prices based on different categories of news articles using multiple kernel learning", Decision Support Systems, 2016, pp. 74-83. 